\title{
An IoT Based Water Quality Testing Device: An Approach to Modelling a Geographical Map Based on Water Quality Data and Decision Support System
}

\author{
Md. Riad Hassan ${ }^{1}$, Pallab Podder ${ }^{2}$, Tarun Debnath ${ }^{3}$, Md. Omar Faruk ${ }^{4}$ \\ ${ }^{1}$ Department of Information and Communication Engineering, Pabna University of Science and Technology, Bangladesh, \\ Email: riad_hassan@outlook.com \\ ${ }^{2}$ Department of Information and Communication Engineering, Pabna University of Science and Technology, Bangladesh, \\ Email: pallabcsu@gmail.com (Corresponding Author) \\ ${ }^{3}$ Department of Information and Communication Engineering, Pabna University of Science and Technology, Bangladesh, \\ Email: iamtarun09@gmail.com \\ ${ }^{4}$ Department of Information and Communication Engineering, Pabna University of Science and Technology, Bangladesh,
} Email: fom_06@yahoo.com

\begin{abstract}
According to the World Health Organization, 30\% people lack access to safe water. Normally water quality is evaluated in a scientific laboratory which is expensive, time-consuming and impractical for real-time implementation. Thus, researchers have introduced different electronic devices to test water quality in real-time. However, these methods focused only on testing device development rather than disseminating resultant information for real-life applications. Moreover, these devices did not have any correlation with mobile decision support system and notification. To address these limitations, an integrated decision support system has been developed for instantaneous water quality detection by hardware configuration, smartphone application and web-based mapping. The evolved device automatically calculates six types of water parameter values, while the smartphone application categorizes water quality and notifies its consumption suitability by decision support system. The smartphone application also stores the GPS location of the water source and water quality data and also sends to the server for generating geographical map concerning its quality. Smartphone application's stored data and central stored data are synchronized based on location. The smartphone also generates notification even without any access to the internet when a user enters into a lowerwater quality region. This package of solutions can be implemented all over the world for ensuring safe water.
\end{abstract}

Key words: Water Quality, Water Testing Device, Decision Support System, Notification System.

\section{INTRODUCTION}

Water quality evaluation is a prominent research area since its application is primarily related to human health as well as environment. The adult male body is composed of about $60 \%$ water while, this figure for an adult woman is about $55 \%$. The amount of water in the human brain, lungs, muscles, kidneys, skin and bones is $73 \%, 83 \%, 79 \%$ and $64 \%$ and $31 \%$ respectively [1]. As water is highly contiguous to our digestive system, impure water increases the degree of contamination and possibility of water-borne diseases. For daily needs in Bangladesh, 62.47 liter per of water per person is required [2]. This quantity for other developed country is higher, for example, about 100 liters per person per day in Australia [3]. Among $71 \%$ of the world water, only $2.5 \%$ is fresh, while in developing countries, more than $50 \%$ people are without access to the fresh water source [4]. So, we need to select pure water source before uses, however, it is impractical to test in laboratory all of these sources and its parameter such as Total Dissolved Solids (TDS), salinity, turbidity, Dissolved Oxygen (DO), $\mathrm{pH}$ and temperature.

Water conducts electricity when salts and different ions dissolve in it [5]. This conductivity is determined by measuring the concentration of the ions or salts dissolved or vice versa [6].This simple method is used to determine the TDS, salinity, turbidity, $\mathrm{DO}, \mathrm{pH}$ and temperature using electronic sensors [7]-[13]. This implementation has been devised by some researchers to determine water quality.

The authors in [14]proposed a model to monitor drinking water quality using IoT. They designed and implemented a circuit with Arduino Uno and sensors to determine temperature and TDS and sent it through internet to show reproduced value. The authors of [15] introduce a microcontroller sensor-based system in which water quality is monitored using IoT in real time to measure water quality parameters such as temperature, $\mathrm{PH}$, turbidity and conductivity. The authors of [16] introduce a device to measure water quality parameter such as temperature, $\mathrm{pH}$, TDS, turbidity by using smart sensor. This implementation is absolutely hardware based and lacks other additional user-friendly features. The authors of [17] introduce a device which can measure $\mathrm{pH}$, TDS, Temperature and only display the result in an LCD display. The main limitations of the existing implementations are: there is no structured way for 
data storage, no option for mobile notification and no geographical mapping opportunity for web-based data access for direct notification about water quality of a region.

The authors of [18] proposed a model of real time water quality monitoring and notification system which is capable to measure temperature, $\mathrm{pH}$ and conductivity. The notification depends on current reading of the sensor and it provides an audio beep alert when water quality parameter values reach at an unsafe level. Moreover, there is no room to store data for visualization and mapping to depict the whole scenario of water quality of an area.

The author of [19] proposed a method of IoT based water Monitoring System where $\mathrm{pH}$, TDS, and Turbidity is measured and data are sent to the cloud server to show values only. There is no offline data display and notification option available in this process.

The authors of [20] introduce a system to monitor contamination of water to avoid water borne diseases by determining $\mathrm{pH}$, turbidity, temperature and sent notification to the third party by SMS services. The authors of [21] introduce water quality monitoring system using IoT which measure only $\mathrm{pH}$ of water and sent SMS to the authority if the value of $\mathrm{pH}$ exits a predefined threshold value. The future work of the last paper is to send the sensor data to cloud for global monitoring of water quality and visualization graphically.

The overall limitations of all the existing water quality related contributions found in the literature can be summarized as follows.

i)Everybody needs to have access to the testing device toknow about water quality;

ii)No decision-support mechanism is involved in the process;

iii) There is no well-structured system for storing the tested data results;

iv)No geographical mapping is exposed based on water quality which is active in webserver to ensure the availability of necessary data to the general people even who does not have any testing device;

v)There is no notification system if anyone stay in a lowerquality water zone when internet in not available.

In this work, we overcome all the above-mentioned limitations to ensure the sources of fresh water for all people. To address the existing limitations, we have developed a hardware device, a smartphone application, cloud data storage and graphical visualization process through geographical mapping based on sored water quality data. In hardware measurement device, sensors for measuring water quality parameters i.e. TDS, salinity, turbidity, DO, pH and temperature are connected with Arduino Uno. When sensors' probe is inserted into water, it provides analog value of the parameters processed by Arduino and calculates TDS, salinity, turbidity, DO, $\mathrm{pH}$ and temperature in their standard units. Then these values are sent to the smartphone application by Bluetooth connection. The smartphone application receives those values as a bit stream [22]. Then it converts the bit stream into 'US-ASCII' character set. Then the corresponding water quality values are represented through colored bar level indicator from green to red- meaning safe to danger level respectively. As a part of decision support, the standard deviation was calculated and the tested result was normalized. Finally, we not only calculate average of normalized deviation but also categorize them based on this average and demonstrate results in smartphone application. Beside this, smartphone application also calculates GPS location and sends all the tested results and GPS location data to the central web server with proper credentials to authenticate and store in its local database. Once authentication is successfully completed, data are stored in server's database. The server dynamically generates geographical map according to the stored value and it eventually depicts water quality of an area. An option of downloading raw data has been made available to researcher for further analysis. These data are downloadable in different formats such as JavaScript Object Notation (JSON), Comma-Separated Values (CSV) and Portable Document Format (PDF). Beside this, the server is capable of sending data to all smartphone devices based on user location. For this service, when a user moves to a new area, his smartphone application is automatically enabled to notify water quality. It also notifies the nearest better water quality areas if the user is existing in an area having inferior water quality. The entire data receiving and sending schema of this paper is depicted in Figure 1.

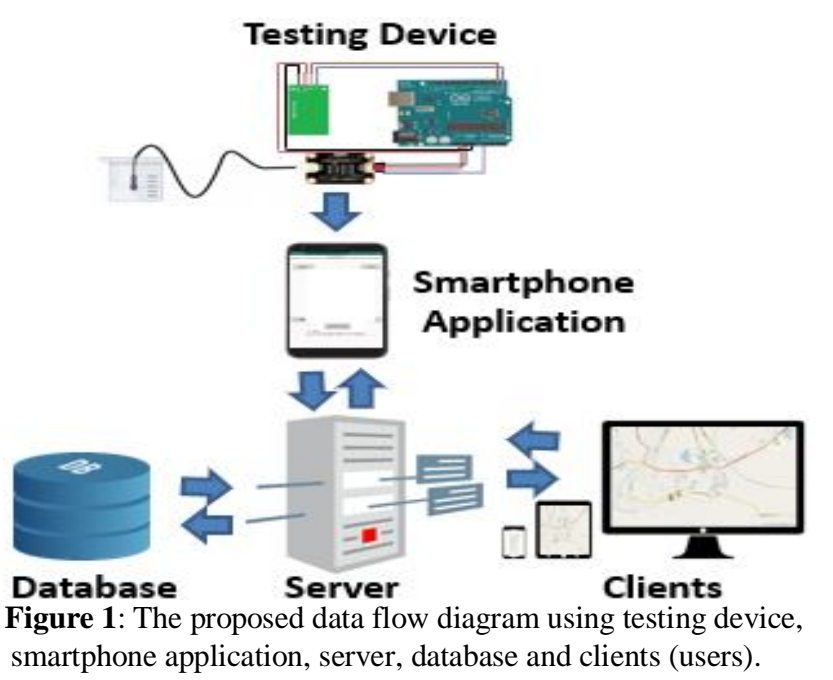

The major contributions of this paper are summarized as follows:

(i) A hardware testing device is developed by which water quality parameters such as TDS, salinity, turbidity, DO, $\mathrm{pH}$ and temperature are calculated.

(ii) A smartphone application is developed for graphical representation of the tested result, local storage and notification.

(iii) The smartphone application users will be notified about the updates during staying at a lower quality water area and provided better quality water source locations even without having continuous access to the internet.

(iv) Water is categorized based on the deviation from standard value of water quality related parameters. 
(v) Central data storage system is developed where data from all mobile apps is stored and all data are open for researcher for further analysis.

(vi) Web based geographical map is developed where water quality data is represented based on the diversified sources of water quality.

(vii) Anyone without testing device will get all data of water quality and would be notified by simply using the proposed smartphone application.

The remainder of the paper is organized as follows: Section 2 illustrates all the key steps for the measurement of water quality, data storage, data synchronization, notification and decision support system; Section 3 broadly represents the data analysis phase; while Section 4 concludes the paper.

\section{PROPOSED IMPLIMENTATION}

The contribution of the proposed method is comprised of three main parts. The testing device configuration, smartphone application and web server development. The entire process is presented as a process diagram in Figure 2 and the key steps are detailed in the following subsections. Note that the contributory parts of this work are marked by dotted green rectangle.

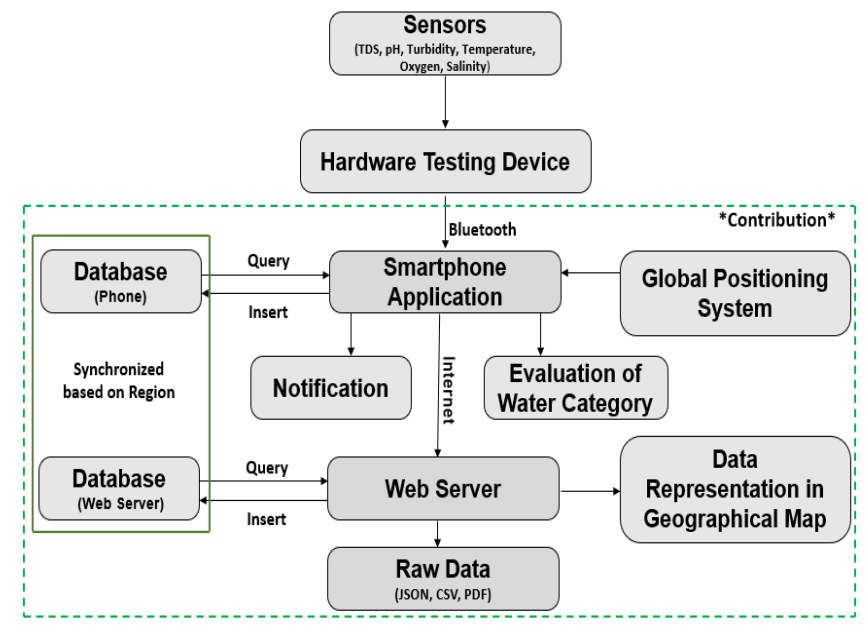

Figure 2: Process diagram of the proposed method for entire system of water testing, categorization of water, tested results data storage, notification and dynamic mapping.

\subsection{Hardware Configuration}

When The hardware device is developed to measure TDS, $\mathrm{pH}$, turbidity, salinity, DO and temperature to determine quality of water andArduino Uno is used as the central processing unit of this device. It is also possible to integrate those sensors with smartphone, however, as the smartphone is not usually water resistant, an additional hardware device is proposed in this regard. The proposed hardware circuit diagram is illustrated in Figure 3.

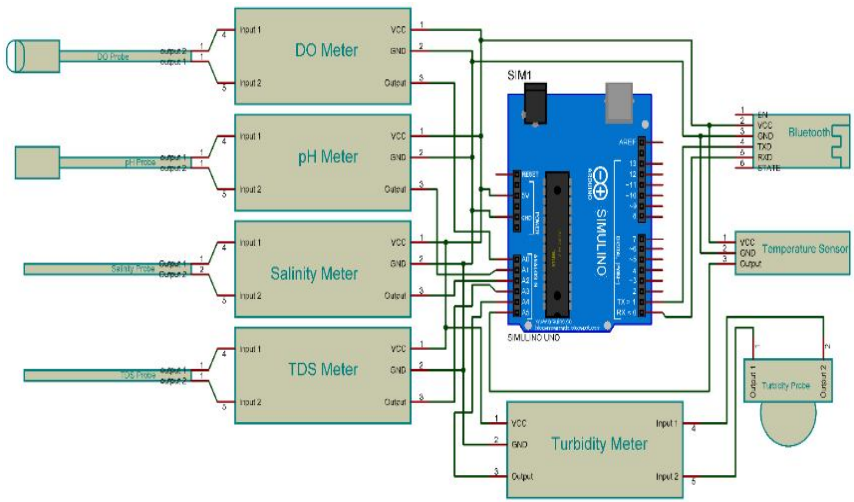

Figure 3: Circuit diagram of water quality testing device that can measure TDS, $\mathrm{pH}$, turbidity, salinity, $\mathrm{DO}$ and temperature of water instantly.

As the sensors are analog in nature, they provide the corresponding analog values of water quality. Then those value are processed using Arduino built-in processor to determine the parameters of water quality. Different sort of measurement processes are equated as follows.

Temperature Measurement: The value getting from temperature sensor is represented as $\beta$. Now analog value converted into voltage level $\mathrm{N}$ in $0-5 \mathrm{~V}$ as source voltage is $5 \mathrm{~V}$.

$$
\mathrm{N}=\frac{\beta \times 5}{1024} \text { volt }
$$

Now, the temperature $\alpha$ is,

$$
\alpha=(\mathrm{N} \times 100)^{\circ} \mathrm{C}
$$

Turbidity Measurement: The value getting from turbidity sensor is represented as $\Theta$. Now analog value converted into voltage level, \& in $0-5 \mathrm{~V}$ as our source voltage is $5 \mathrm{~V}$.

$$
\S=\frac{\Theta \times 5}{1024} \text { volt }
$$

Now, the turbidity of water $\mathrm{T}$ in Nephelometric Turbidity Units (NTU) is

$$
T=3 \times\left(-1120 \oiint^{2}+5720 \oiint-4352.9\right) N T U
$$

pH Measurement: The value getting from $\mathrm{pH}$ sensor is represented as $(x)$. Now analog value converted into voltage level $\mathrm{b}$ in $0-5 \mathrm{~V}$ as our source voltage is $5 \mathrm{~V}$.

Now, the $\mathrm{pH}$ is

$$
\zeta=\frac{() \times 5}{1024} \text { volt }
$$

$$
p H=-18.75 \wp-15.53
$$

TDS measurement: The value is taken from TDS sensor once in duration $\mathrm{t}$ millisecond. If we input $\mathrm{n}$ analog values, the values are

$$
\begin{array}{r}
\mathrm{A}_{(\mathrm{TDS})}=\left\{\mathrm{A}_{1}, \mathrm{~A}_{2}, \mathrm{~A}_{3} \ldots \ldots \ldots \mathrm{A}_{\mathrm{n}}\right\} \\
A_{n}=\left\{\begin{array}{cl}
a_{n} & T \text { mod } t=0 \\
\text { null } & \text { otherwise }
\end{array}\right.
\end{array}
$$

Where,

$A_{n}=n t h$ value from the TDS sensor.

$t=$ duration

$a=$ TDS sensor value.

$T=$ Timestamp in millisecond 
For getting more appropriate value by the median filtering algorithm

$$
A_{\text {med }}=\operatorname{median}\left[\operatorname{sort}_{\text {ascending }}\left(A_{n}\right)\right]
$$

Where, $A_{\text {med }}=$ Filtered stable value.

Now the selected analog value is represented as the voltage value in $0-5 \mathrm{~V}$ as our source voltage is $5 \mathrm{~V}$.

$$
€=\frac{A_{\text {med }} \times 5}{1024} \text { volt }
$$

Where, $I$ I = A convert to voltage level

Here, another factor temperature plays an important role to change TDS of water. The TDS value depends on temperature. So, we should compensate the TDS value with respect to the temperature.

Now the compensation coefficient is,

$$
\mathrm{h}=1+0.02(\alpha-25)
$$

Where,

$\mathrm{b}=$ Compensation coefficient.

$\alpha=$ Temperature in degree Celsius.

Now the compensated voltage is,

$$
\zeta=\frac{\digamma}{\mathrm{h}} \text { volt }
$$

Where,$\zeta=$ Compensated Voltage.

At last the now the TDS value will be

$$
T D S=\frac{133.42 z^{3}-255.86 z^{2}+857.39 z}{2} \mathrm{mg} / \mathrm{l}
$$

Salinity Measurement: If the received value from salinity sensor is $\Omega$, salinity of that water is

$$
\text { Salinity }=(\Omega / 1950)^{-\frac{1}{0.87}} \mathrm{mg} / \mathrm{l}
$$

DO Measurement: The value is taken from DO sensor once in duration $\mathrm{t}$ millisecond. If we take $\mathrm{n}$ analog value, the values are,

$$
\begin{gathered}
\mathrm{B}_{(\mathrm{DO})}=\{\mathrm{B} 1, \mathrm{~B} 2, \mathrm{~B} 3 \ldots \ldots . \mathrm{Bn}\} \\
B_{n}=\left\{\begin{array}{cc}
a & \text { Tmod } t=0 \\
\text { null } & \text { otherwise }
\end{array}\right.
\end{gathered}
$$

Were,

$$
\begin{array}{ll}
\mathrm{B}_{\mathrm{n}}=n t h \text { value from } \mathrm{DO} \text { sensor } & a=\mathrm{DO} \text { sensor value } \\
T=\text { Timestamp in millisecond } & t=\text { duration }
\end{array}
$$

For getting more appropriate value by the median filtering algorithm

$$
B_{\text {med }}=\operatorname{median}\left[\operatorname{sort}_{\text {ascending }}\left(B_{(D O)}\right)\right]
$$

Where,

$$
B_{\text {med }}=\text { Filtered stable value } \text {. }
$$

Now the selected analog value is represented as the voltage value.

$$
\varnothing=\frac{B_{\text {med }} \times 5}{1024} \times 1000 \mathrm{mV}
$$

Now, the dissolved oxygen, DO is

$$
D O=\frac{1127.6 \times \varnothing}{8.24} \mathrm{mg} / \mathrm{l}
$$

The resultant values of the equations are relative and depend on device components, source voltage, device calibration and water sources. Other related equations may work better, however, the proposed implementation suits best when compared to the ground truth reference of each parameter.

\subsection{Smartphone Application}

Smartphone application receives water quality parameter values from hardware device (as discussed in the previous section 2.1) using Bluetooth connection. The prime feature of this application is that it enables a user to get notification and the tested result from central server even without having any testing device. Smartphone app is a collection of activity and services [23]. This application comprises three activities and two services which are broadly shown in Figure 4.

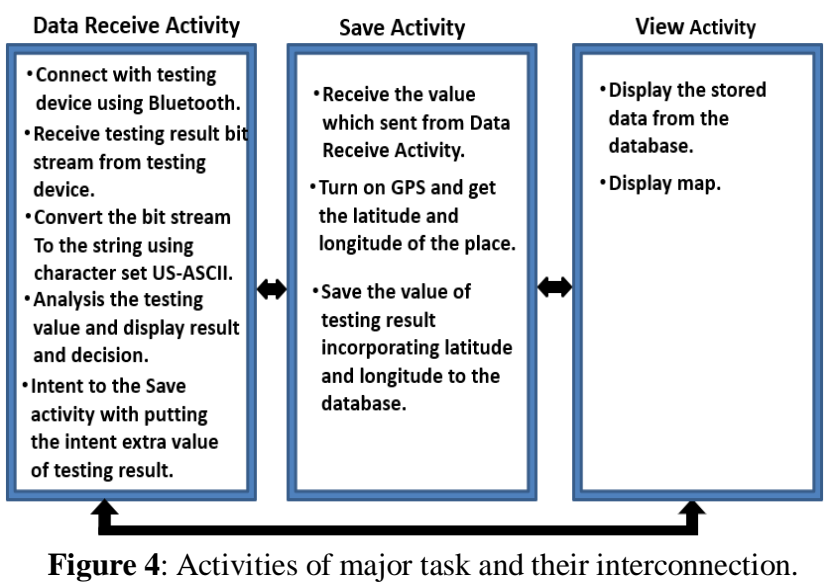

First one is Data Receive Activity which is responsible for Bluetooth connection between the proposed hardware device and mobile phone to receive data. After successful establishment of connection, the data exchange process begins. To this end, water quality measurement data are sent to the smartphone from hardware device as a bit stream and then bit streams is converted into character set 'US-ASCII'. Now the calculated values of water quality are represented by bar colored level indicator where green to red indicates safe to danger quality respectively. Beside this, we calculate the deviation of every tested water quality parameter value from its standard value. For TDS, Turbidity, DO has a fixed threshold level. If the tested value is lower than threshold (€), water is safe to drink in this regard.

The $\mathrm{pH}$, salinity and temperature do not have any fixed threshold like other parameters. However, those parameters have a safe range of values and any value beyond this range is evidently unsafe. For such range of values, the mid-point is considered as standard value. So, if range of lower point is $\mu$ and upper limit is $\eta$, standard value $€$ (typical threshold) will be,

$$
€=\frac{\eta-\mu}{2}
$$

Considering the tested value $\Phi$, the deviation $\phi$ of water quality parameters (TDS, Turbidity, DO, pH, Salinity, Temperature) will be,

$$
\phi=|€-\Phi|
$$

For calculation of deviation, the standard water parameter values from the World Health Organization (WHO) and Bangladesh government have been compared prior to the final insertion in Table 1[24]-[25]. 
Table 1: Standard value of different water quality parameter.

\begin{tabular}{|l|l|}
\hline Parameter & Acceptable level \\
\hline Total dissolved Solids (TDS) & $<600 \mathrm{mg} / \mathrm{l}$ \\
\hline Turbidity & $<5 \mathrm{NTU}$ \\
\hline Dissolved Oxygen (DO) & $6.5-8 \mathrm{mg} / \mathrm{l}$ \\
\hline $\mathrm{pH}$ & $6.5-8.5$ \\
\hline Salinity & $<180 \mathrm{mg} / \mathrm{l}$ \\
\hline Temperature & $25-40^{0} \mathrm{C}$ \\
\hline
\end{tabular}

Now, calculate the normalized deviation $\boldsymbol{\gamma}$ with respect to standard value $€$,

$$
\gamma=\frac{\phi}{€}
$$

Now, find the average normalized deviation of all six parameters (TDS, $\mathrm{pH}$, turbidity, salinity, DO, temperature) and it is denoted as quality factor $\varepsilon$,

$$
\varepsilon=\frac{1}{6} \sum_{i=1}^{6} \gamma_{i}
$$

The quality indicator $\varepsilon$ is such a factor which helps categorize water into different levels. Water category with respect to quality factor is given in Table 2 .

Table 2: Categorization of water according to the quality factor $\varepsilon$

\begin{tabular}{|l|l|}
\hline Quality factor, $\varepsilon$ & Water category \\
\hline 0.00 & Ideal \\
\hline $0.01-0.04$ & Good \\
\hline $0.05-0.09$ & Average \\
\hline $0.10-0.14$ & Poor \\
\hline $0.15-0.19$ & Very Poor \\
\hline $0.20-0.24$ & Bad \\
\hline$\geq 0.25$ & Dangerous \\
\hline
\end{tabular}

Next activity is Save Activity which is responsible to save the data and determine GPS location. It receives water quality parameter data from Data Receive Activity and determines GPS location using built-in smartphone's GPS sensor. Those data are saved in SQLite database of the application. The third activity is the View Activity which is responsible for viewing the saved data and mapping with local database. Two services run background of the application and serve the purpose. The first service is the synchronization of application database with web server database which is to be discussed in section 2.3 , while the second one is treated as notification which is to be described in section 2.4 .

\subsection{Database synchronization}

When water is tested, the resultant data in accordance with water source GPS location is stored in smartphone database and initially marked as not sent as this data is not already sent to the central server. When the smartphone is connected with internet, the data is immediately sent to central database of web server and marked as sent in the smartphone database. In this way data from all smartphone application are populated in central server. On the other hand, when a user enters a new area which water quality data is not available his smartphone application, it automatically requests with GPS location of that area for sending water quality data. In response to this request, central server sends all data of water quality of $1 \mathrm{Km}^{2}$ area considering provided GPS location as center position. Smartphone application saves those received data in its database.

\subsection{Notification}

Smartphone gets water quality data of any area in data synchronized process described in section 2.3. In this way necessary data become available for every smartphone automatically. Getting current GPS location from build-in GPS sensor, smartphone application compares the location with stored water quality data and generate notification for dangerous area. One position is considered as a danger area if water category is lower than a user specified threshold within 100 -meter radius from that point. The notification also contains the nearest safe water source locations.

\subsection{Web Server}

The main features of the web server are the central storage of all water quality data coming from smartphone applications, provide on demand data for notification generation as described in section 2.3 and 2.4. It also generates water quality based geographical map dynamically for users who have neither testing device nor smartphone so that they can aware in a simple way by browsing map from web browser. To develop this web service, we use Semantic UI framework as a frontend and Bottle for backend [26]. Bottle is a python web micro framework [27]. A well featured python package named Folium is used for rendering geographical map which depict scenario of water quality. Water category is considered ideal by representing Deep-Green, good by Light-Green, average by Orange, poor by Pink, very poor by Purple, bad as Black and dangerous as Red color marker in the geographical map. By hovering on marker, anyone can see the category of water and by clicking marker of the map can get specific value of that particular point's TDS, pH, salinity, DO, turbidity and temperature. To serve such purpose, we use three routes in our web service which are 'result', 'rawData' and 'dataRecieve'.

\begin{tabular}{|c|c|c|}
\hline @route(/dataRecieve) & @route(/rawDat) & @route(/result) \\
\hline $\begin{array}{l}\text { - Authenticate HTTP PUT } \\
\text { request. } \\
\text { - Get HTTP PUT request } \\
\text { parameter values. } \\
\text { - Validate values. } \\
\text { - Store the data in the } \\
\text { database. }\end{array}$ & $\begin{array}{l}\text { • Handle HTTP GET } \\
\text { request } \\
\text { - Purse all data in } \\
\text { different format } \\
\text { (CSV, PDF, Jason) }\end{array}$ & $\begin{array}{l}\text { - Get data from } \\
\text { database. } \\
\text { - Generate map } \\
\text { for client }\end{array}$ \\
\hline
\end{tabular}
Key functions of each route are depicted in Figure5.

Figure 5: Function of different route of the web Service

The first one of the routes is used for representing dynamically generated map, second one for representing all raw data which are downloadable in different format such as 
PDF, CSV, JSON and the last one is for receiving data from smartphone application. In data receiving phase, the web server authenticates the smartphone application's request through secret key which is intergraded with smartphone application. This step protects our sever from false requests.

The whole operation of this project is presented in following algorithm.

\section{Algorithm:}

1. Input analog value corresponding water quality in Arduino using sensor and calculate water quality parameters value in standard unit.

2. Sent the values to smartphone application through Bluetooth.

3. Receive bit stream data of water quality in smartphone application from Arduino and convert from bit stream to 'US-ASCII' character set.

4. Calculate deviation from standard value of all water quality parameter and normalize.

5. Take average of normalized deviation of water quality and classify water.

6. Depict the tested result value graphically as well as classification of water in smartphone and store tested result data in local storage of smartphone application.

7. Sent tested result data to web server through HTTP request.

8. Authenticate HTTP request in web server and store in database and data send to mobile application based on area.

9. Dynamically generate geographically map based on water quality and render data in different format such as JSON, CSV, PDF.

10. Data are synchronized between smartphone application and central web server based on location.

11. Notify users when users enter in lower quality water area and provide nearest higher quality water source location.

\section{EXPERIMENTAL DATA ANALYSIS}

This experiment has been conducted in every division of Bangladesh and its North, South, East and West districts. Moreover, some exceptional places such as costal, low-land, high-land, hilly and proximity to beach are also taken into consideration. Beside this, we also consider river water as some people depend on river as water source. Thus, we select 40 regions water source and marked them 1 to 40 randomly. To conduct this experiment, we collect water sample from all the selected areas and insert testing device probe into water. The sensors of the device sense corresponding water quality parameter (i.e. TDS, $\mathrm{pH}$, turbidity, salinity, DO, temperature) values and then those values are converted into associated units as explained earlier in section 2.2.Tested result data are graphically represented in figure6(a-f) to analyze which values are in accepted portion. Figure $6(a-b)$ are in logarithm scale where logarithm base is 10 so that data visualization becomes clear. Figure 6(c-f) are depicted using normal
scaling.Green shade area of figure6(a-f) indicates acceptable value of corresponding water quality parameter.

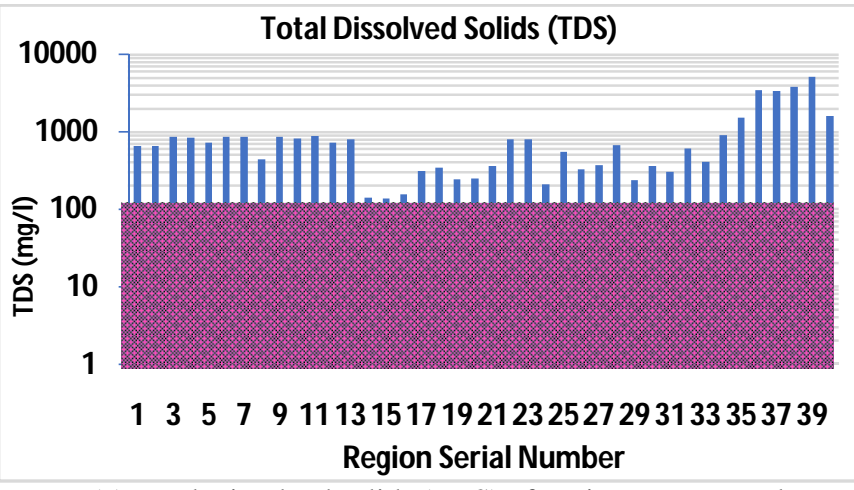

(a) Total Dissolved solids (TDS) of testing water samples

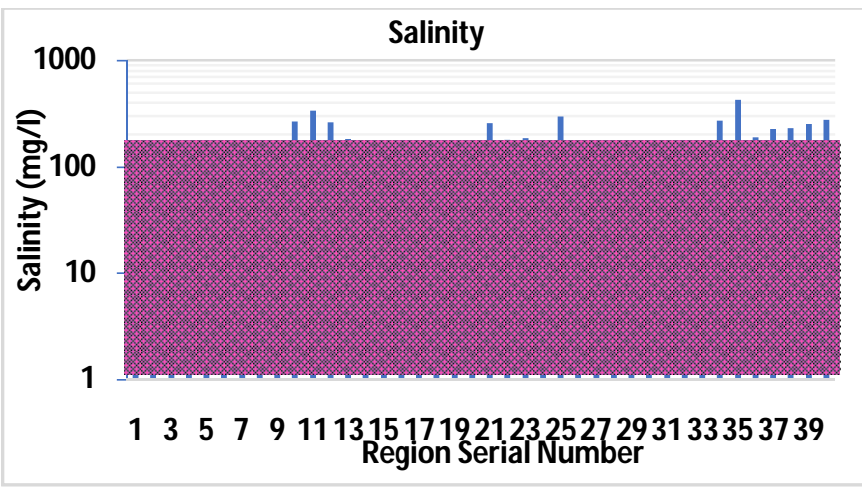

(b) Salinity of testing water samples

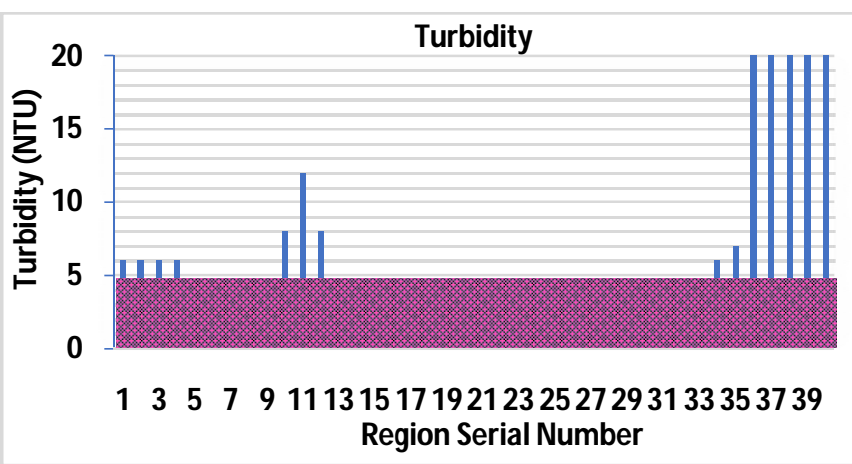

(c) Turbidity of testing water samples

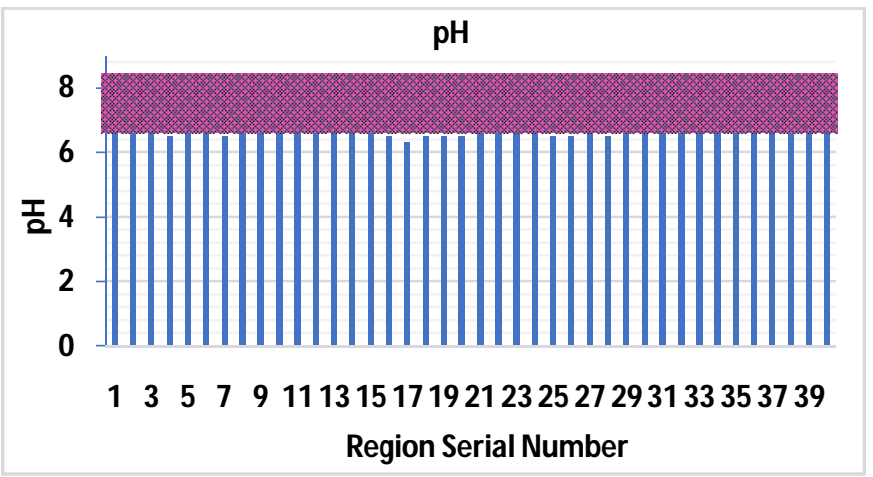

(d) $\mathrm{pH}$ of testing water samples 


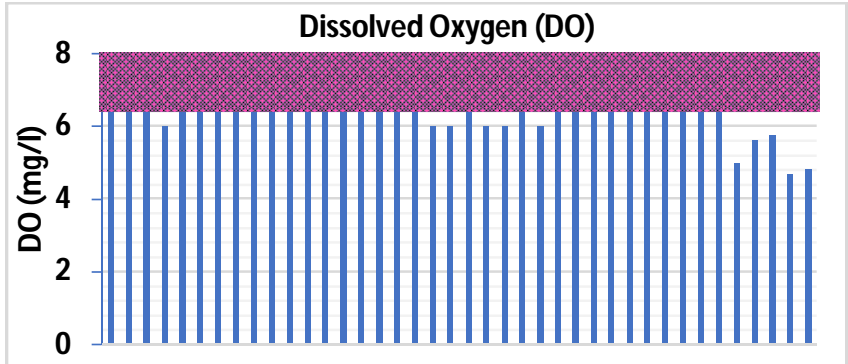

13579111315171921232527293133353739 Region Serial Number

(e) Dissolved Oxygen (DO) of testing water samples

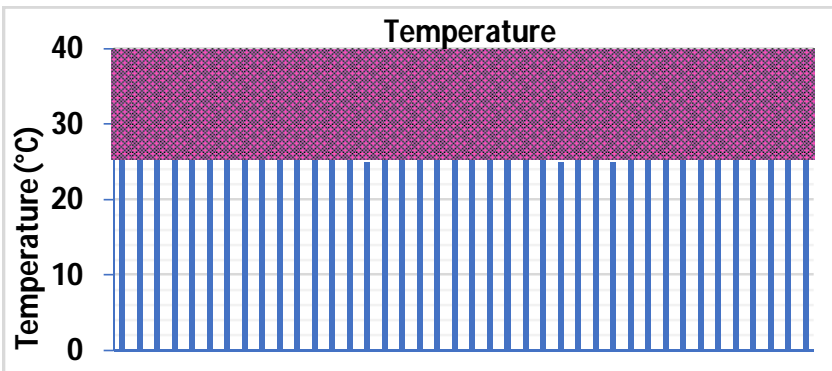

\section{9 Region Serial Number}

(f) Temperature of testing water samples

Figure6: Graphical representation of six water quality (TDS, Salinity, Turbidity, pH, DO, Temperature) of all samples. Here, the value of water quality parameters in green shade area is acceptable.

From figure6, we observe that most of water quality parameters' value of region serial number $10,11,12,35,36$, 37, 38, 39, 40 not in acceptable range.

In each and every testing time, those six water quality parameter values are received by Smartphone application from hardware testing device. It determines deviation from the standard value of water quality parameters, normalizes it and then categorizes water for user decision support system as explained earlier in section 2.2.The calculation of quality factorto categorize water source data is shown in figure 7.

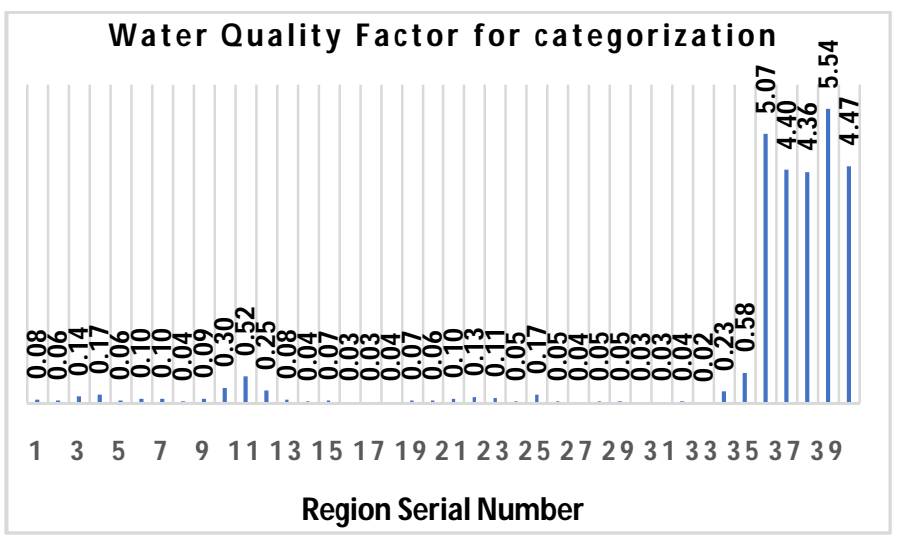

Figure 7: Water quality factor for categorization of water source from measured water quality parameters.

From figure 7 we can observe 10, 11, 12, 35, 36, 37, 38, 39, 40 no water source quality factor, $\varepsilon<0.25$. So, they are marked as Dangerous. In same way other sources also marked.
During execution phase of the experiment, screenshot of smartphone application in different stages is depicted in figure 8(a-d). Here, serial no. 1 data is considered as we first took those screen shorts as an experimental.
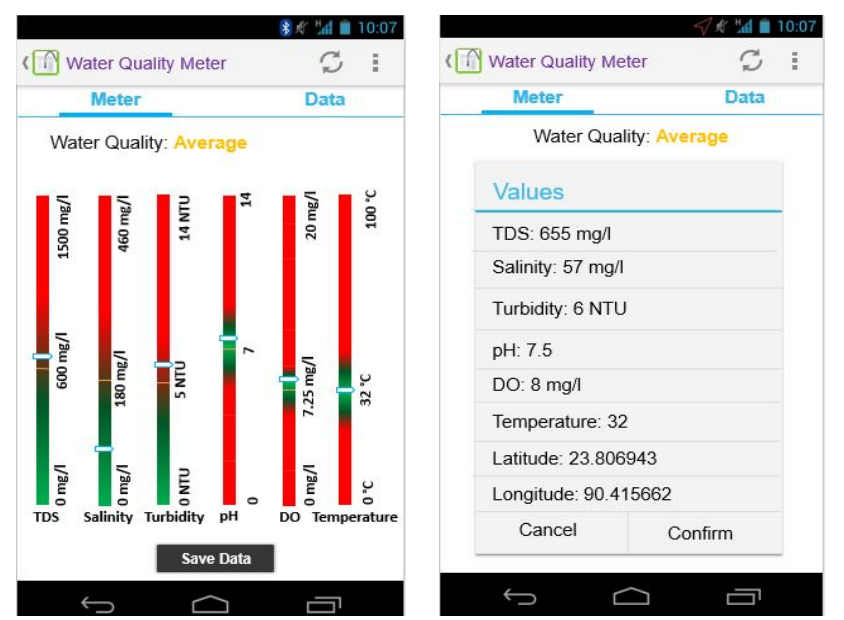

(a) Water testing result getting from hardware testing device indicate in Data Receive Activity of app on bar level where green is safe and red is not safe.

(b) Display data which intent from Data Receive Activity and calculate GPS location in Save Activity of app for save in database.

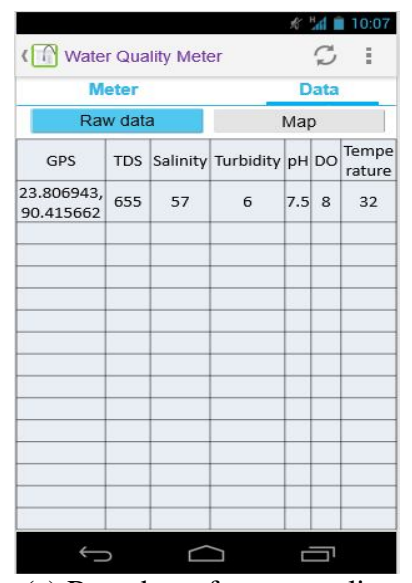

(c) Raw data of water quality parameter in View Activity of app.

Figure 8: Screen short of smartphone application's different activity at the time of first experiment

When data was received from hardware testing device, it was data displayed on corresponding scale of water quality parameters as shown in figure 8-a. After pressing the 'Save Data' button, the 'Data Receive Activity' intent those six values to 'Save Activity' stage. The 'Save Activity' receives the values as well as calculates GPS location and stores it in the database show as shown in figure 8-b. The stored data are displayed in raw form and map as presented in figure 8-c and figure 8-d respectively.

From smartphone application all the tested data combined with GPS location are stored in central server's database. Using those data, central server generate map which demonstrates the water quality with respect to the GPS 
location points. Getting data from central server's database, the web map view that appeared in the browser is presented in figure 9 where different color of markers demonstrate different categories of water source in the map.

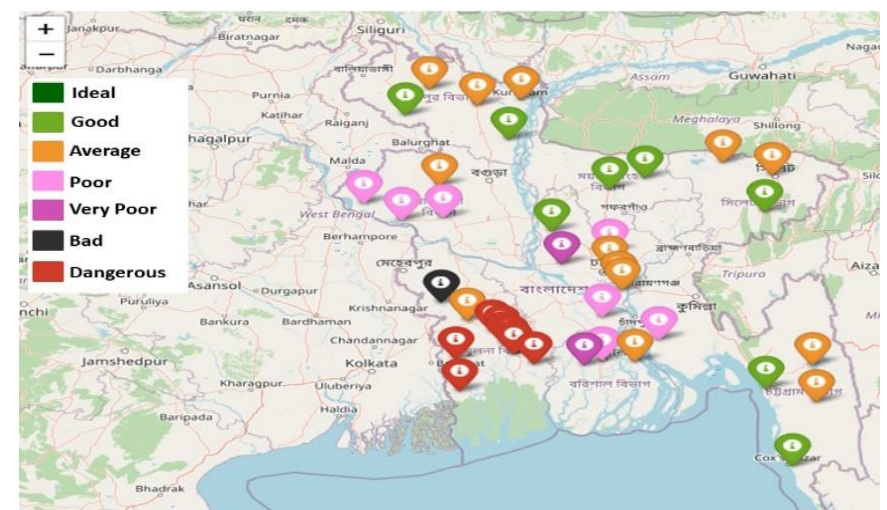

Figure9: Dynamically generated geographical map which depicts water quality.

The application of the proposed implementation has been confined for the context of Bangladesh which however can be extended continent-wise. When all the data of the continents are accumulated in a single framework (a lengthy project indeed), water quality data would be available to all the conscious people regardless of considering the locations.

\section{CONCLUSION}

World Health Organization states three in every ten persons do not get proper water for drinking, even most of them do not aware about their drinking water quality because of the complexity of water testing. Normally, the quality of water is assessed in a scientific laboratory that is expensive, time-consuming and also impractical for real-time implementation. So, researchers introduce sensor based portable electronic device so that anyone can test water at anytime and anywhere. however, such techniques concentrated only on the development of testing device rather than disseminating the findings for real time applications. The testing device is capable only to show water quality parameter value that is not understandable to all the general user. To address these limitations, we develop a complete package of solution that can support to take decision of user for selecting drinking water. To do this we develop a hardware water testing device which can determine six water quality parameter (TDS, salinity, turbidity, $\mathrm{pH}, \mathrm{DO}$, temperature) instantly, smartphone application which uses as a decision support system as well as it can notify users when they are in danger zone of water, central web server which stores all tested result data centrally and dynamically generates geographical map based on water quality. At first water is tested by hardware testing device and tested result values are sent to smartphone. Smartphone determine deviation from standard value of corresponding water quality parameter and normalize. Based on the mean of normalized values, smartphone application categorizes the quality of water and display to user as a decision support system. Beside this, smartphone application calculates water source GPS location using its build in GPS sensor and store in local storage of smartphone as well as sends to central web server to store centrally. Data are synchronized based on location between smartphones and central server so that necessary data are available in smartphone to provide proper alerts even without internet connectivity. The system can be applied across the world to ensure proper water in every sector, especially for drinking and helping to lead healthy life

\section{ACKNOWLEDGEMENT}

This work was supported in part by the ICT Division, Ministry of Posts, Telecommunications and Information Technology, People's Republic of Bangladesh under Innovation Grant in Information and Communication Technology. Tracking ID 19IF12654.

\section{REFERENCES}

1. How Much of Your Body Is Water? What Percentage?[Online].Available:https://www.thoughtco. com/how-much-of-your-body-is-water-609406. [Accesse d: 21-Oct-2019].

2. A. H. Milton, H. Rahman, W. Smith, R. Shrestha, and K. Dear,Water consumption patterns in rural Bangladesh : are we underestimating total arsenic load ?, J. Water Health, vol. 04.4, pp. 431-436, 2006.

3. Riverina Water, Average Water Use. [Online]. Available:https://www.rwcc.nsw.gov.au/savewater/aver age-water-use. [Accessed: 17-Oct-2019].

4. K. S. Adu-Manu, C. Tapparello, W. Heinzelman, F. A. Katsriku, and J. D. Abdulai, Water quality monitoring using wireless sensor networks: Current trends and future research directions, ACM Transactions on Sensor Networks, vol. 13, no. 1. Association for Computing Machinery, 01-Jan-2017.

5. E. D. Goddard and G. C. Benson, CONDUCTIVITY OF AQUEOUS SOLUTIONS OF SOME PARAFFIN CHAIN SALTS, Can. J. Chem., vol. 35, no. 9, pp. 986-991, Sep. 1957.

6. T. Lambrou, C. Anastasiou, C. Panayiotou, and M. Polycarpou, A LowCost Sensor Network for Real-Time Monitoring and Contamination Detection in Drinking Water Distribution Systems, IEEE Sensors Journa, vol. 14, no. 8, pp. 2765-2772, 2014.

7. R.WAGNER, Guide to Environmental Analytical Methods, 4th editio. Genium Publishing Corporation, Schenectady, 1998.

8. E. A. Atekwana, E. A. Atekwana, R. S. Rowe, D. D. Werkema, and F. D. Legall, The relationship of total dissolved solids measurements to bulk electrical conductivity in an aquifer contaminated with hydrocarbon,J. Appl. Geophys., vol. 56, no. 4, pp. 281-294, 2004.

9. A. Hyldgård, D. Mortensen, K. Birkelund, O. Hansen, and E. V. Thomsen, Autonomous multi-sensor micro-system for measurement of ocean water 
salinity, Sensors Actuators, A Phys., vol. 147, no. 2, pp. 474-484, Oct. 2008.

10. A. Bin Omar and M. Bin MatJafri, Turbidimeter Design and Analysis: A Review on Optical Fiber Sensors for the Measurement of Water Turbidity,Sensors, vol. 9, no. 10, pp. 8311-8335, Oct. 2009.

11. D. Xiao, Y. Mo, and M. M. F. Choi, A hand-held optical sensor for dissolved oxygen measurement, Meas. Sci. Technol., vol. 14, no. 6, p. 862, May 2003.

12. D. K. Maurya, A. Sardarinejad, and K. Alameh, High-sensitivity pH sensor employing a sub-micron ruthenium oxide thin-film in conjunction with a thick reference electrode, Sensors and Actuators, A: Physical, vol. 203. Elsevier B.V., pp. 300-303, 01-Dec-2013.

13. Y. Zhao and Y. Liao, Novel optical fiber sensor for simultaneous measurement of temperature and salinity, Sensors Actuators, B Chem., vol. 86, no. 1, pp. 63-67, Aug. 2002.

14. Y. Al-Khashab, R. Daoud, M. Majeed, and M. Yasen, Drinking Water Monitoring in Mosul City Using IoT, 2019, pp. 1-5.

15. N. Vijayakumar and R. Ramya, The real time monitoring of water quality in IoT environment,ICIIECS 2015 - 2015 IEEE Int. Conf. Innov. Information, Embed. Commun. Syst., 2015.

16. Taufiqurrahman, N. Tamami, D. A. Putra, and T. Harsono, Smart sensor device for detection of water quality as anticipation of disaster environment pollution, in Proceedings - 2016 International Electronics Symposium, IES 2016, 2017, pp. 87-92.

17. K. Indu and J. J. Choondal, Modeling, development \&amp; analysis of low cost device for water quality testing, in 2016 IEEE Annual India Conference (INDICON), 2016, pp. 1-6.

18. N. A. Cloete, R. Malekian, and L. Nair, Design of Smart Sensors for Real-Time Water Quality Monitoring,IEEE Access, vol. 4, pp. 3975-3990, 2016.
19. P. Damor and K. J Sharma, IoT based Water Monitoring System: A Review, Int. J. Adv. Res. Sci. Eng., vol. 4, no. 6, 2017.

20. K. C. of E. and T.Karpagam(Department of Computer Science and Engineering et al., Real Time Water Contamination Monitoring System using IoT to avoid Water borne diseases in Textile Cluster Areas, Int. J. Adv. Res. Sci. Eng., vol. 07, no. special-02, pp. 228-235, 2018.

21. N. R. Moparthi, C. Mukesh, and P. Vidya Sagar, Water qualiy monitoring system using IOT, in Proceedings of the 4th IEEE International Conference on Advances in Electrical and Electronics, Information, Communication and Bio-Informatics, AEEICB 2018, 2018.

22. W. Ren and Z. Miao, A hybrid encryption algorithm base on DES and RSA in Bluetooth communication, in Proceedings - 2010 2nd International Conference on Modeling, Simulation, and Visualization Methods, WMSVM 2010, 2010, pp. 221-225.

23. S. Holla and M. M. Katti, ANDROID BASED MOBILE APPLCATION DEVELOPMENT and its SECRITY, 2012.

24. M. Kumar and A. Puri, A review of permissible limits of drinking water, Indian Journal of Occupational and Environmental Medicine, vol. 16, no. 1. Wolters Kluwer -- Medknow Publications, pp. 40-44, Jan-2012.

25. WHO, Guidelines for drinking-water quality, 4th edition, incorporating the 1st addendum. WHO, 2017.

26. O. Khriyenko, Semantic UI: Automated Creation of Semantically Personalized User Interface, 2015.

27. R. Arai, S. Sato, and H. Iwasaki, A debugger-cooperative higher-order contract system in Python, in Lecture Notes in Computer Science (including subseries Lecture Notes in Artificial Intelligence and Lecture Notes in Bioinformatics), 2016, vol. 10017 LNCS, pp. 148-168. 